\title{
Flocculation of MXenes and their use as 2D Particle Surfactants for Capsule Formation
}

Huaixuan Cao, ${ }^{1, \dagger}$ Maria Escamilla, ${ }^{2, \dagger}$ Kailash Dhondiram Arole, ${ }^{1}$ Dustin Holta, ${ }^{2}$ Jodie L. Lutkenhaus, ${ }^{1}$ Miladin Radovic, ${ }^{2}$ Micah J. Green, ${ }^{1,2 *}$ Emily B. Pentzer ${ }^{2,3 *}$

${ }^{1}$ Artie McFerrin Department of Chemical Engineering, Texas A\&M University, College Station, TX USA ${ }^{2}$ Department of Materials Science and Engineering, Texas A\&M University, College Station, TX USA

${ }^{3}$ Department of Chemistry, Texas A\&M University, College Station, TX USA

$\dagger$ These authors contributed equally

*Corresponding authors

Table S1. $\zeta$ potential for $\mathrm{Ti}_{3} \mathrm{C}_{2} \mathrm{~T}_{\mathrm{z}}$ MXenes

Figure $\mathrm{S} 1$. Transmission electron microscopy (TEM) image of $\mathrm{Ti}_{3} \mathrm{C}_{2} \mathrm{~T}_{\mathrm{z}}$ nanosheets.

Figure S2. Optical microscopy images of $\mathrm{Ti}_{3} \mathrm{C}_{2} \mathrm{~T}_{\mathrm{z}}$ emulsions with no salt addition

Figure S3. Optical microscopy images for high concentrations of $\mathrm{Ti}_{3} \mathrm{C}_{2} \mathrm{~T}_{\mathrm{z}}$

Figure S4. Time study: $\mathrm{NaCl}$-flocculated $\mathrm{Ti}_{3} \mathrm{C}_{2} \mathrm{~T}_{\mathrm{z}}$ stabilized emulsions

Figure S5. Time study: $\mathrm{Ti}_{3} \mathrm{C}_{2} \mathrm{~T}_{\mathrm{z}}$ stabilized emulsions flocculated with different salts $(0.0025 \mathrm{M})$

Figure S6. MXene emulsions with high concentrations of $\mathrm{LiCl}$ and its time study

Figure S7. Optical microscopy images and corresponding droplets size analysis for emulsions and capsules stabilized by $\mathrm{NaCl}$ flocculated $\mathrm{Ti}_{3} \mathrm{C}_{2} \mathrm{~T}_{\mathrm{z}}$ MXenes.

Figure S8. Thermogravimetric analysis (TGA) of neat polyurea polymer shell and $\mathrm{NaCl}$ flocculated $\mathrm{Ti}_{3} \mathrm{C}_{2} \mathrm{~T}_{\mathrm{z}}$ capsules

Figure S9. Fourier transform infrared (FTIR) spectroscopy of MXene capsules formed from the flocculation with $\mathrm{NaCl}$ and $\mathrm{CsCl}$

Figure S10. High resolution X-Ray photoelectron spectroscopy (XPS) data (C 1s, N 1s, O 1s) of NaCl flocculated $\mathrm{Ti}_{3} \mathrm{C}_{2} \mathrm{~T}_{\mathrm{z}}$ capsules

Figure S11. Energy dispersive X-ray spectroscopy (SEM-EDS) of $\mathrm{NaCl-flocculated} \mathrm{Ti}_{3} \mathrm{C}_{2} \mathrm{~T}_{\mathrm{z}}$ capsules

Figure S12. Optical microscopy images, scanning electron microscopy (SEM), and X-Ray photoelectron spectroscopy (XPS) of capsules synthesized with $\mathrm{CsCl}$ as the flocculating agent. 
Table S1. $\zeta$ Potential for $\mathrm{Ti}_{3} \mathrm{C}_{2} \mathrm{~T}_{\mathrm{z}}$ nanosheets at different $\mathrm{NaCl}$ concentrations.

\begin{tabular}{|l|l|l|l|l|l|l|}
\hline $\mathrm{NaCl}$ concentration (M) & 0 & 0.005 & 0.025 & 0.05 & 0.1 & 0.5 \\
\hline $\boldsymbol{\zeta}$ Potential (mV) & -46 & -51.2 & -50.5 & -39.4 & -28 & -14.1 \\
\hline
\end{tabular}
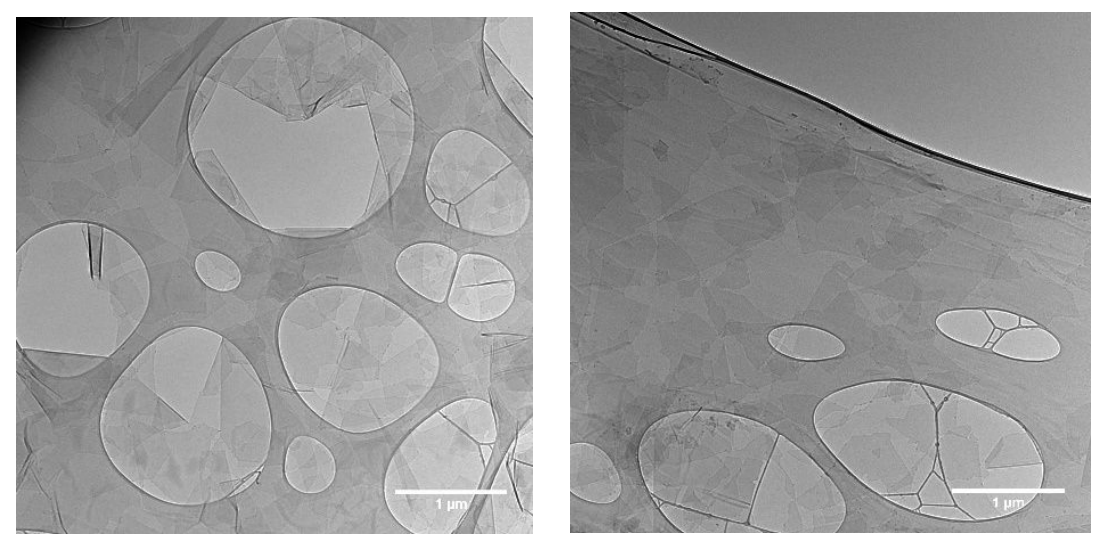

Figure S1. Transmission electron microscopy images of $\mathrm{Ti}_{3} \mathrm{C}_{2} \mathrm{~T}_{\mathrm{z}}$ nanosheets.

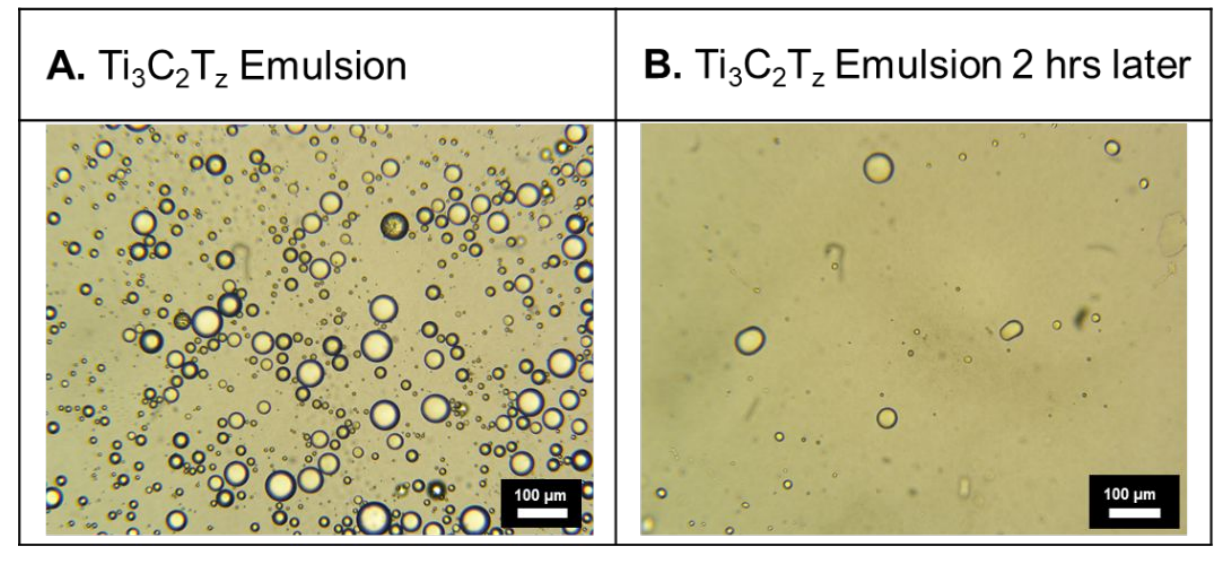

Figure S2. (A) Neat $\mathrm{Ti}_{3} \mathrm{C}_{2} \mathrm{~T}_{\mathrm{Z}}$ stabilized emulsion and (B) 2 hrs after the emulsion was prepared. 


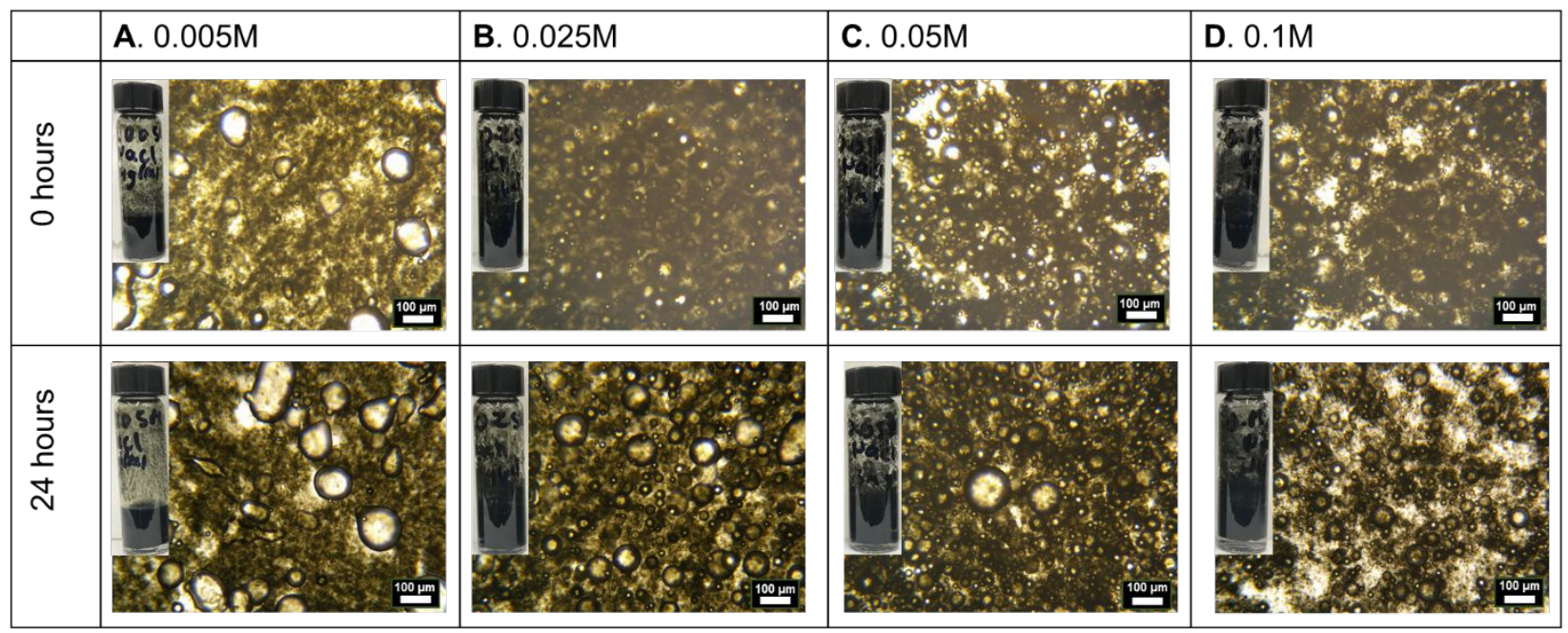

Figure S3. Optical microscopy images and photographs of 1:5 ratio toluene-in-water emulsions stabilized by flocculated $3 \mathrm{mg} / \mathrm{mL} \mathrm{Ti}_{3} \mathrm{C}_{2} \mathrm{~T}_{\mathrm{z}}$ nanosheets using $\mathrm{NaCl}$ at $\mathrm{A}$ ) $0.005 \mathrm{M}, \mathrm{B}$ ) $\left.0.025 \mathrm{M}, \mathrm{C}\right) 0.05 \mathrm{M}$, and D) $0.1 \mathrm{M}$.

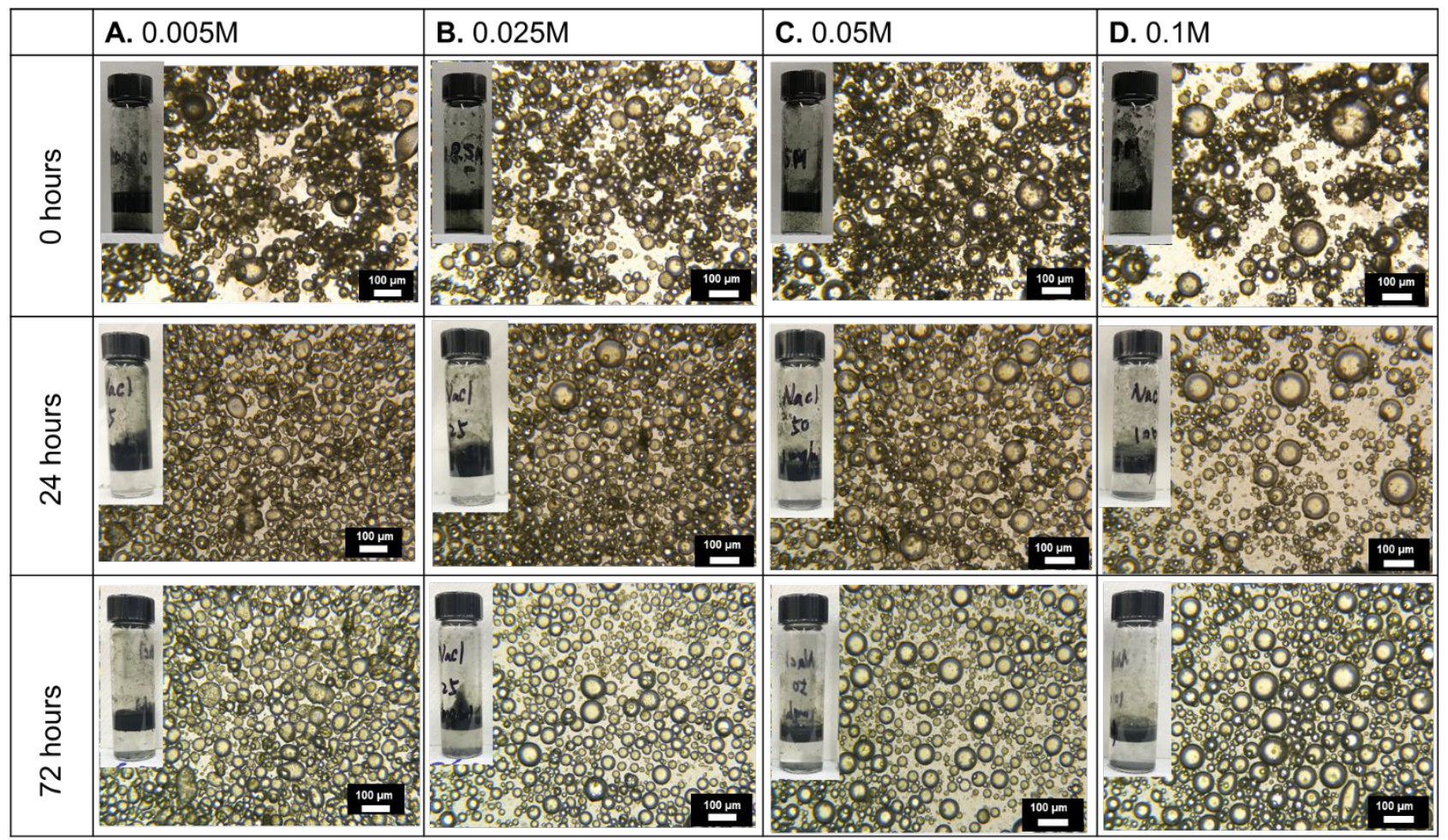

Figure S4. Stability time study - Optical microscopy images and photographs of 1:5 ratio toluene-in-water emulsions stabilized by flocculated $1 \mathrm{mg} / \mathrm{mL} \mathrm{Ti}_{3} \mathrm{C}_{2} \mathrm{~T}_{\mathrm{z}}$ nanosheets using $\mathrm{NaCl}$ at A) $0.005 \mathrm{M}, \mathrm{B}$ ) $0.025 \mathrm{M}, \mathrm{C}$ ) $0.05 \mathrm{M}$, and D) $0.1 \mathrm{M}$. 


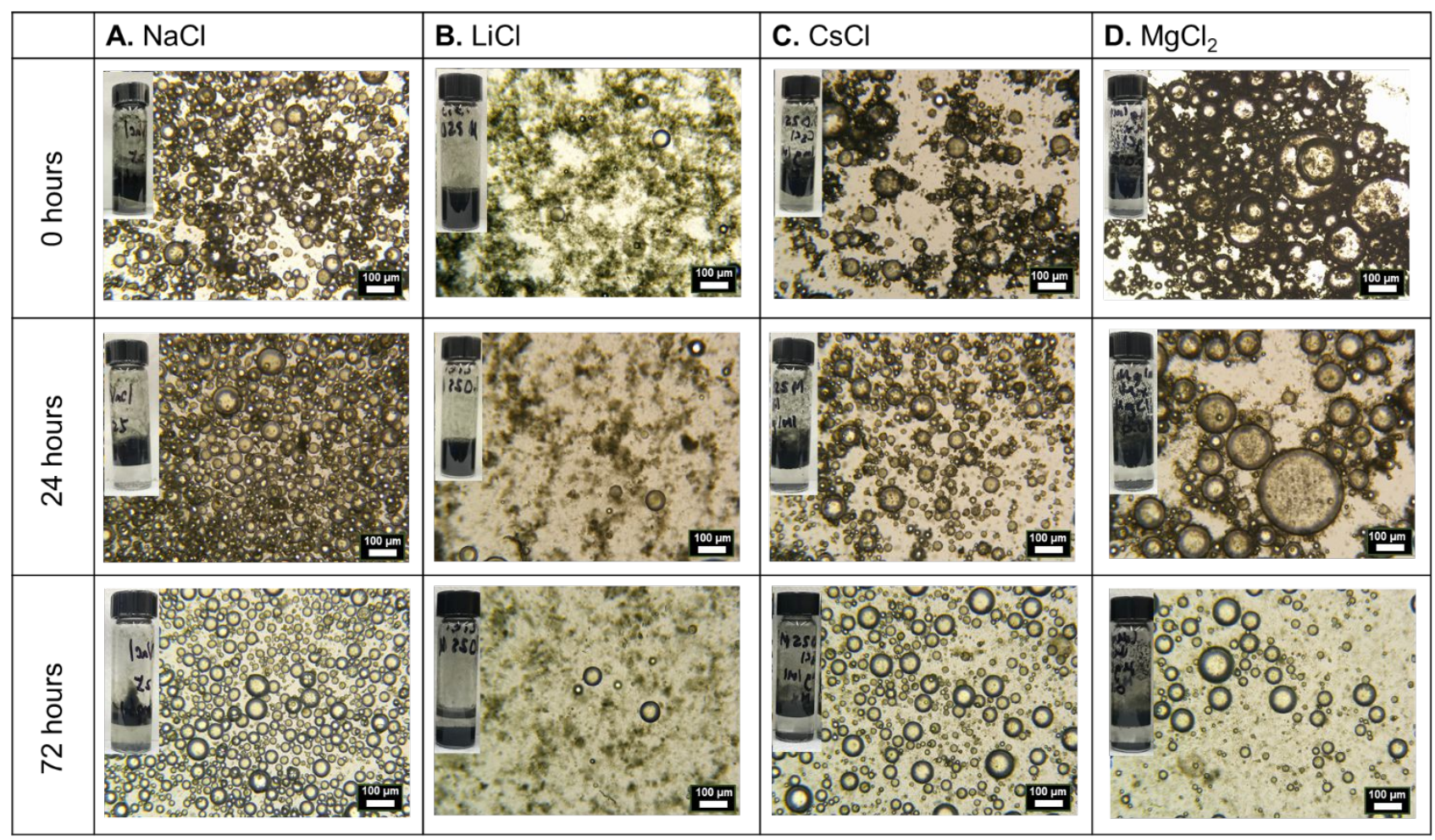

Figure S5. Stability study overtime - MXene toluene-in-water emulsions at $0.025 \mathrm{M}$ concentrations for (A) $\mathrm{NaCl}$, (B) $\mathrm{LiCl}$, (C) $\mathrm{CsCl}$, and (D) $\mathrm{MgCl}_{2}$ at $0 \mathrm{hrs}, 24 \mathrm{hrs}$, $72 \mathrm{hrs}$ kept at room temperature. 


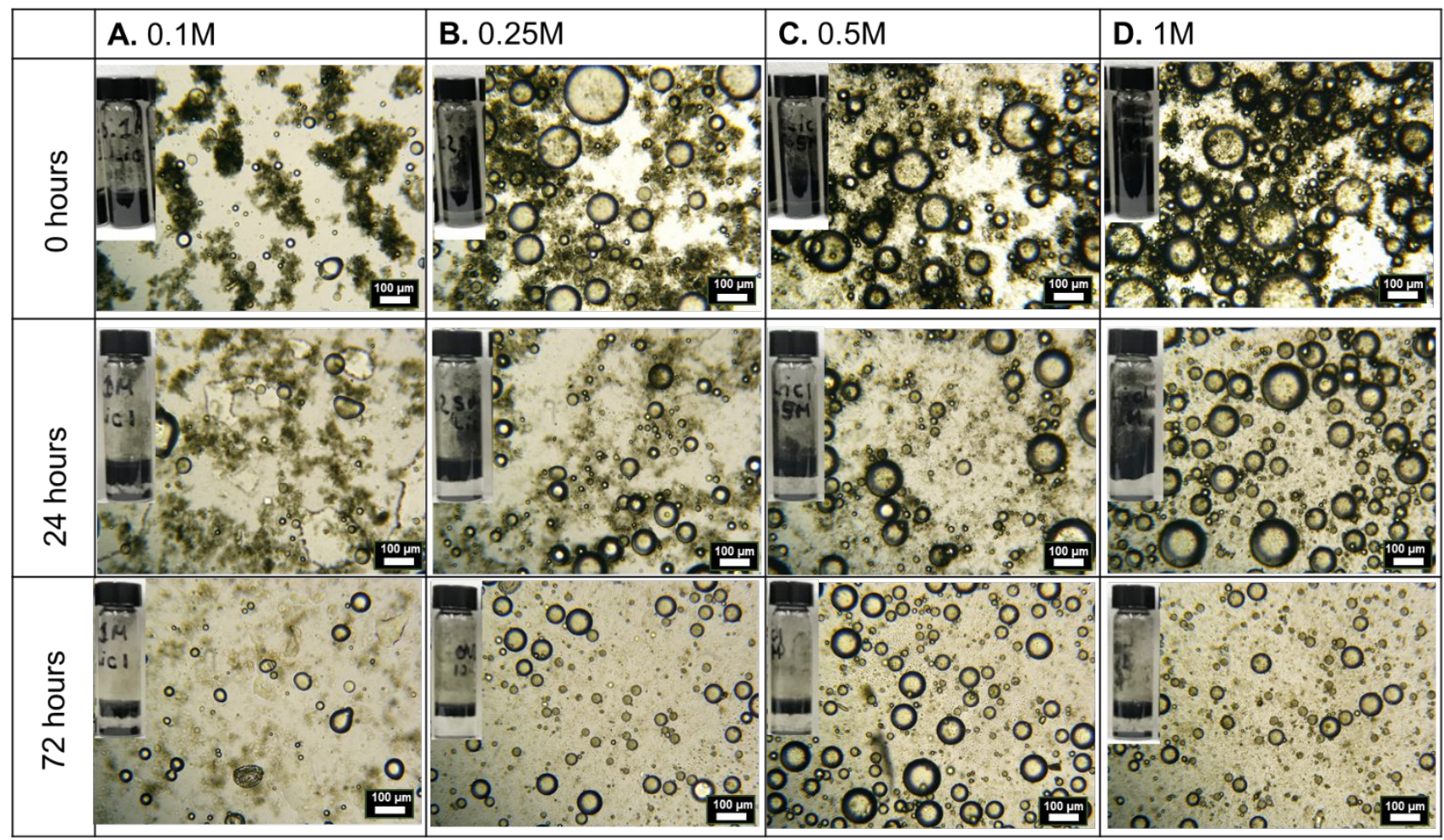

Figure S6. Stability time study - Optical microscopy images and photographs of 1:5 ratio toluene-in-water emulsions stabilized by flocculated $1 \mathrm{mg} / \mathrm{mL} \mathrm{Ti}_{3} \mathrm{C}_{2} \mathrm{~T}_{\mathrm{z}}$ nanosheets using $\mathrm{LiCl}$ at $\left.\left.\mathrm{A}\right) 0.1 \mathrm{M}, \mathrm{B}\right) 0.25 \mathrm{M}, \mathrm{C}$ ) $0.5 \mathrm{M}$, and D) $1 \mathrm{M}$.
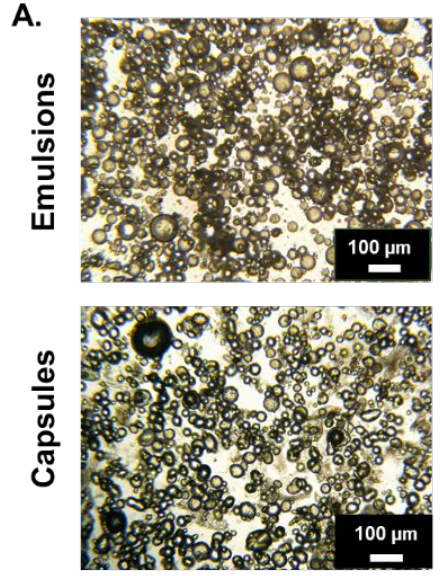
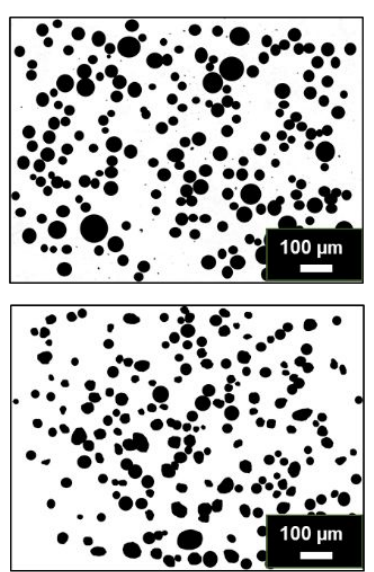

B.

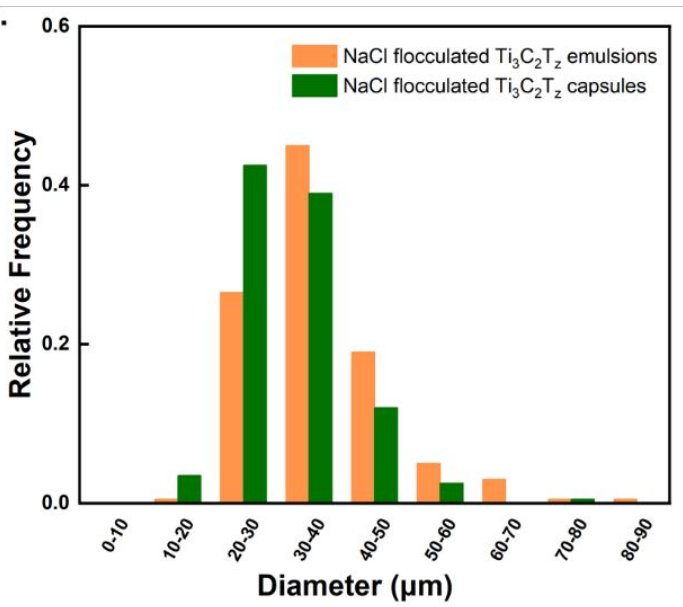

Figure S7. A) Optical microscopy images and corresponding droplet outlines for size analysis of the emulsions and capsules stabilized by $\mathrm{NaCl}$-flocculated $\mathrm{Ti}_{3} \mathrm{C}_{2} \mathrm{~T}_{\mathrm{z}}$ nanosheets; $\mathrm{B}$ ) size distribution of the emulsion droplets and capsules stabilized by $\mathrm{NaCl}$-flocculated $\mathrm{Ti}_{3} \mathrm{C}_{2} \mathrm{~T}_{\mathrm{z}}$ nanosheets derived from Image $\mathrm{J}$ analysis. 


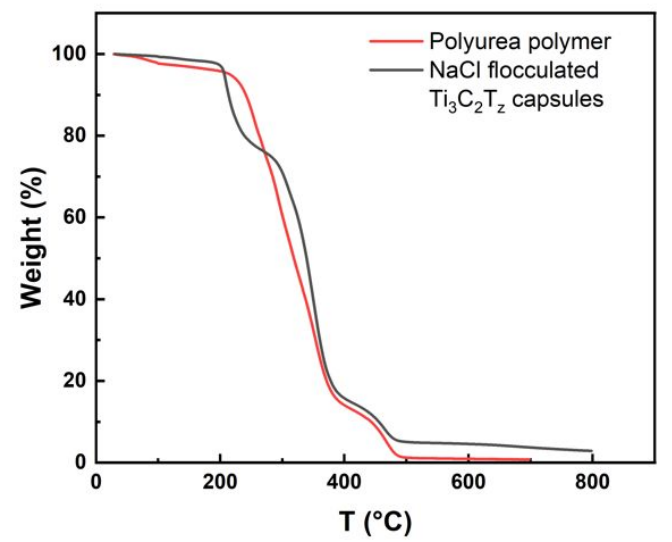

Figure S8. Thermogravimetric analysis (TGA) of neat polyurea polymer shell and $\mathrm{NaCl}$ flocculated $\mathrm{Ti}_{3} \mathrm{C}_{2} \mathrm{~T}_{\mathrm{z}}$ capsules

A.

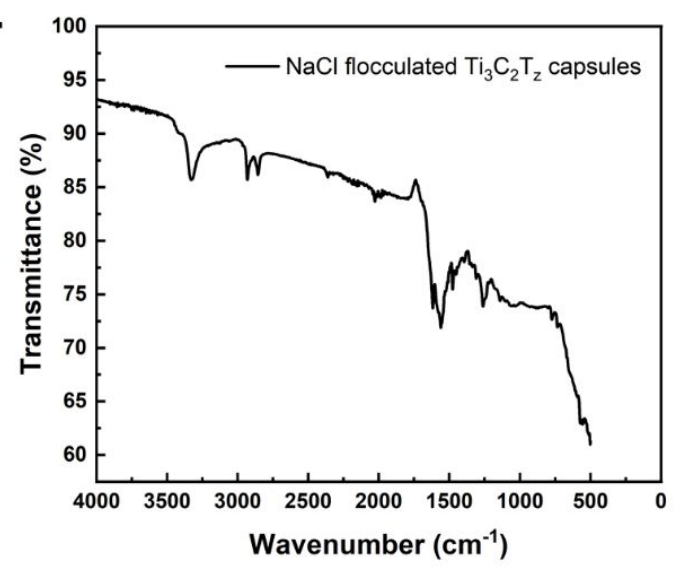

B.

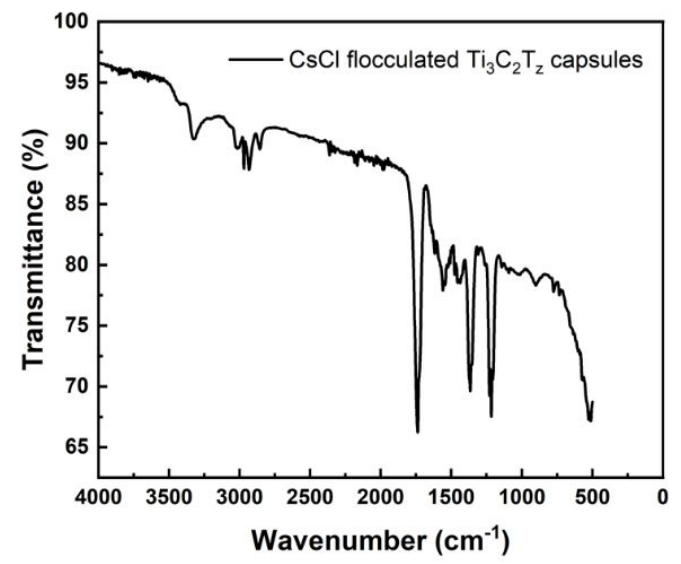

Figure S9. Fourier transform infrared (FTIR) spectroscopy of $\mathrm{Ti}_{3} \mathrm{C}_{2} \mathrm{~T}_{\mathrm{z}}$ capsules formed from the flocculation with (A) $\mathrm{NaCl}$ and (B) $\mathrm{CsCl}$. 

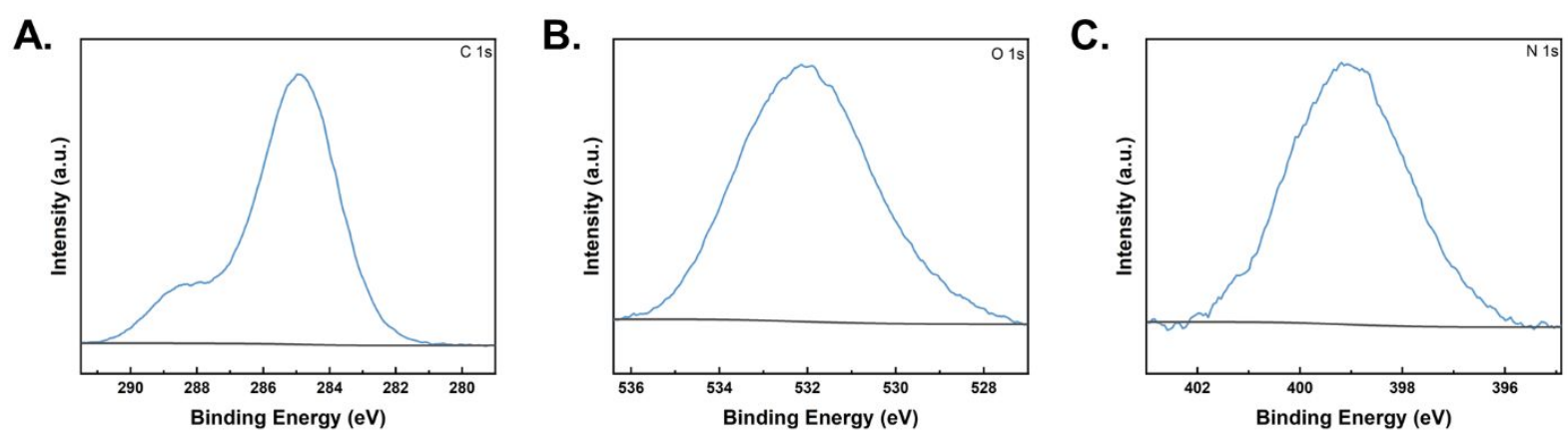

Figure S10. High resolution XPS data of C1s N1s O1s of $\mathrm{NaCl}$ flocculated $\mathrm{Ti}_{3} \mathrm{C}_{2} \mathrm{~T}_{\mathrm{z}}$ capsules
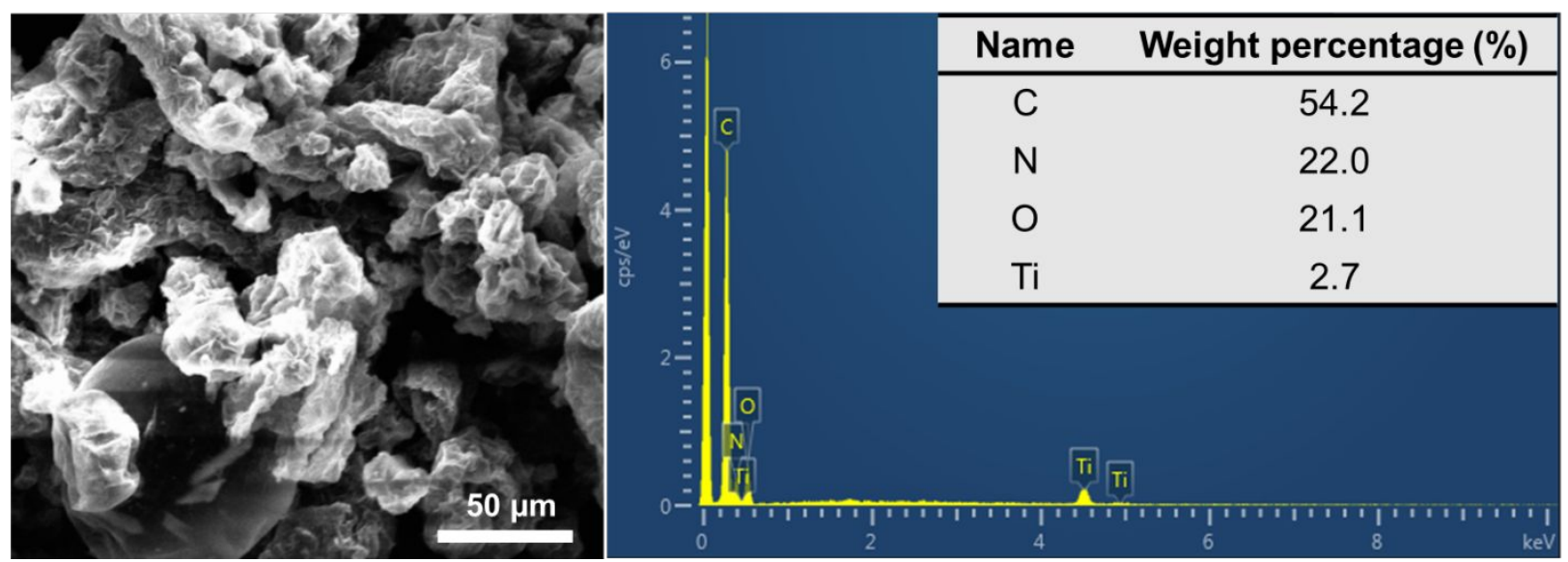

Figure S11. Energy dispersive X-ray spectroscopy (SEM-EDS) of $\mathrm{NaCl}$ flocculated $\mathrm{Ti}_{3} \mathrm{C}_{2} \mathrm{~T}_{\mathrm{z}}$ capsules 

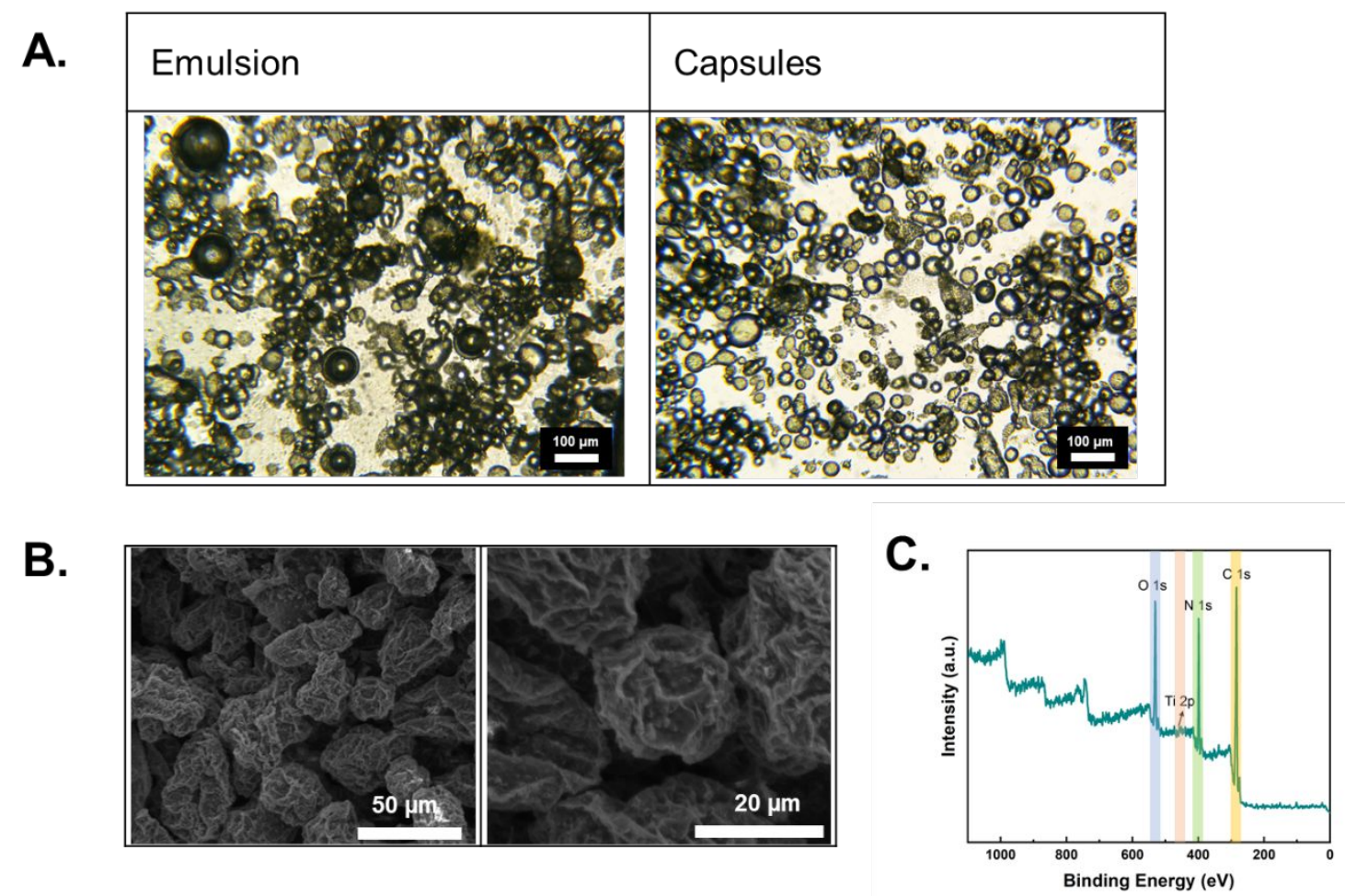

Figure S12. A) Optical microscopy of HDI toluene-in-water $1 \mathrm{mg} / \mathrm{mL} \mathrm{Ti}_{3} \mathrm{C}_{2} \mathrm{~T}_{\mathrm{z}}$ emulsion and crosslinked capsules using $0.005 \mathrm{M} \mathrm{CsCl}$. B) SEM images of vacuum dried capsules. C) XPS of $\mathrm{CsCl}$ capsules 